\title{
Estudio inicial de dos Programas para la formación De Licenciados en Química
}

\section{Initial Study of two Programs for Chemistry Teachers' \\ TRAINING}

\author{
Rafael Yecid Amador Rodríguez* \\ rafaelamador@uni.pedagogica.edu.co \\ Rómulo Gallego Badillo*** \\ rgallego@uni.pedagogica.edu.co
}

\section{REsumen}

El presente artículo se deriva de un trabajo de investigación realizado en torno a las concepciones epistemológicas, didácticas y pedagógicas que orientan la formación inicial de profesores de química de dos programas de universidades oficiales de Bogotá, Colombia.

Palabras clave: Formación inicial de profesores, ciencias, epistemología, didáctica, pedagogía, currículo y ciencia.

\section{Abstract}

The present paper comes from a research work about the epistemological, didactical and pedagogical conception that leads the chemistry teacher's initial training of two state universities programs in Bogotá, Colombia.

Key words: Science teacher's initial training, Epistemology, Didactics, Pedagogy, Curricula and Science.

* Universidad Pedagógica Nacional.

** Profesor e Investigador de la Universidad Pedagógica Nacional y Coordinador e Integrante del Grupo IREC. Candidato a Magíster en Docencia de la Química. 


\section{INTRODUCCIÓN}

El origen de este artículo es el trabajo de grado realizado por el autor principal (Amador-Rodríguez, 2003) para optar al tí́tulo profesional de licenciado en química. Se decidió estudiar la formación inicial (licenciaturas) de profesores de química ofrecidas por la Universidad Pedagógica Nacional y la Universidad Distrital Francisco José de Caldas, que recibieron acreditación previa obligatoria del Ministerio de Educación Nacional (MEN), de acuerdo con lo dispuesto en el Decreto 272 de 1998 (ya derogado), por lo que se centró en identificar y caracterizar las concepciones epistemológicas, didácticas y pedagógicas que orientan estos programas de formación.

Desde las referencias bibliográficas internacionales (Dumas-Caré, Fruió y Garret, 1990; Furió Mas y Gil Pérez, 1989; Gallego Badillo, Pérez Miranda, y Torres de Gallego, 2002) para ubicar el problema, hay que dejar claro que, en Colombia, la formación inicial de profesores de ciencias sólo se ha desarrollado dentro de programas a cargo de las facultades de Educación y ha sido una formación universitaria distinta de otra carreras. Es necesario señalar que, a partir del Decreto-Ley 080 de 1980, el título para los profesionales de la educación es el de Licenciado; algo que se sugiere ha de marcar la literatura de los fundamentos.

\section{INTENCIONALIDADES}

El estudio inicial busca identificar, a partir del análisis de los documentos de los dos programas, hasta qué punto se inscribieron en la nueva didáctica de las ciencias (Gil, Carrascosa y Martínez Terrades, 1999) desde la perspectiva de la hoy denominada ciencia de enseñar ciencias. Se admite que es un trabajo inicial en vista de que, de conformidad con lo establecido por los especialistas en esta nueva ciencia de enseñar ciencias, es un campo de conocimiento que apenas comienza en Colombia.

\section{Formación inicial y continua de Profesores de Ciencias Experimentales}

La investigación acerca de la formación inicial y continua de profesores de ciencias es un campo de conocimiento en la actual didáctica de las ciencias experimentales (Gil, Carrascosa y Martínez Terrades, 1999), dentro del cual se pueden vislumbrar varias tendencias. Una de éstas pro- pone que la formación ha de girar en torno a lo que ha de saber y saber hacer un docente en ciencias (Gil, 1991).

Un aspecto importante en la formación de profesores en ciencias en esta corriente, es que el docente debe saber la ciencia que enseña; 
por tanto ha de tener un dominio conceptual y metodológico de dicha ciencia. Pero tal dominio no consiste sólo en conocer los principios, teorías y leyes que caracterizan una ciencia, sino que, además, ha de tenerse en cuenta el referente histórico del desarrollo de dicha ciencia, lo cual implica conocer sus procesos de construcción y de evolución (González et al., 1996).

Un programa de formación inicial en el que se contemple el estudio de ese desarrollo histórico-epistemológico ha de proporcionar al futuro docente argumentos para dar cuenta de cómo los científicos participaron en la construcción de dicha ciencia, en este caso la química.

Los resultados de algunas investigaciones (Mellado, 1999) resaltan que ciertos docentes no consideran necesaria una formación didáctica y pedagógica, por lo que manifiestan que basta con saber la ciencia para enseñarla, apelando a su conocimiento de sentido común acerca de cómo enseñar (Gil, 1991); entre ellos hay quienes aducen una pretendida "vocación" como condición suficiente para dicho desempeño, lo que va en contravía del concepto de profesionalidad docente y de la existencia misma de los programas académicos de formación.

En este campo de investigación, los investigadores exponen que la falta de una formación rigurosa en los contenidos de la ciencia por enseñar se refleja en su actividad como docen- tes (Furió, 1994), pues esta debilidad conceptual y metodológica en la disciplina y, en su enseñanza, imponen la repetición y transmisión de los contenidos curriculares (Furió Mas y Gil Pérez, 1989), remitidas exclusivamente a los textos de enseñanza, por lo que practican tal enseñanza esperando de los estudiantes la repetición memorística de tales contenidos curriculares. Este aspecto es uno de los cuestionamientos más significativos que se hacen a los programas de formación de profesores de ciencias en la actualidad.

Uno de los resultados que arrojan las investigaciones en la formación de profesores de ciencias (Furió, 1994) es la poca cercanía de los contenidos presentados en los currículos que orientan el trabajo en clase de los docentes de los programas de formación. Se propone, entonces, como problema de investigación la enseñanza que realizan estos docentes, desplazando la atención investigativa del aprendizaje hacia el ejercicio docente del profesor mismo.

Los investigadores destacan también que, en su práctica docente, los futuros profesores han de integrar su formación disciplinar con la práctica profesional, ya que aquellos que han ejercido la docencia durante varios años no suelen articular la formación académica recibida con su trabajo (Dumas-Carré, Furió y Garret, 1990). Entonces, el abordaje del ejercicio profesional se hace sólo desde unas creencias, ideas, metáforas, actitu- 
des y habilidades, y no desde una posición teóricamente fundamentada; esto es, el enseñar no se realiza bajo unos presupuestos conceptuales y metodológicos, sino desde meras creencias (Furió, 1994). Estas investigaciones resaltan que a los docentes les preocupa más cuánto enseñan de la disciplina que cómo la enseñan, sin tener en cuenta que, desde la didáctica de las ciencias, estos dos aspectos (el conocimiento de la disciplina y su didactización) se deben integrar para favorecer la enseñanza de las ciencias (Furió, 1994).

Hoy se tiene el convencimiento de que quienes ingresan a los programas de formación inicial de profesores de ciencias ya tienen ideas, concepciones y actitudes, elaboradas desde sus vivencias sobre la ciencia que se enseña y el rol de los profesores, que es preciso tener en cuenta (Mellado, 1999) para asumir la formación como un proceso de cambio necesario de dichos saberes previos. De ahí, que en la formación inicial de profesores sea preciso hacer énfasis en la relación inseparable entre la didáctica y la ciencia objeto de enseñanza (Mellado y González, 2000).

Un aspecto relevante en dicho campo son las prácticas docentes que realizan los estudiantes durante su formación inicial, que pretenden integrar la ciencia por enseñar con todo el conocimiento existente acer- ca de su enseñanza. Se propone que estas prácticas constituyan el punto de partida para la construcción teórica del ejercicio profesional del futuro docente (Mellado y González, 2000). Las prácticas de enseñanza conforman el espacio para la reflexión sobre los fundamentos de la profesión, ya que permiten contrastar los fundamentos conceptuales y metodológicos de la profesionalidad. Para tal efecto, estas prácticas han de presentar una orientación y una planificación pues, de lo contrario, se llegaría nuevamente al paradigma tradicional-artesanal en que se admite la formación docente como una modelización del ejercicio (DumasCarré, Furió y Garret, 1990). Esta modelización significa seguir un modelo previamente establecido.

Dicho lo anterior, el objetivo del presente trabajo es la caracterización de las concepciones epistemológicas, didácticas y pedagógicas que orientan la formación inicial de profesores de química en la Universidad Pedagógica Nacional y la Universidad Distrital Francisco José de Caldas. Recientes investigaciones resaltan que en esos programas los profesores de ciencias no requieren una formación didáctica y pedagógica (Gallego y Pérez, 2002) y que basta con saber la ciencia para enseñarla, por lo que se considera que el ejercicio docente se realiza bajo el paradigma de transmisión verbal de contenidos. 


\section{Aspecto Epistemológico general}

En cuanto a la concepción epistemológica, para efectos del estudio, ésta se encaró desde los presupuestos constructivistas, basados en la lógica deductiva, en la que se considera que el conocimiento es una construcción colectiva (Hodson, 1985). Para sostener los presupuestos constructivistas se partió de los planteamientos de Popper (1962), quien rechaza la idea de que el conocimiento se desarrolla a partir de la aplicación de la lógica inductiva. Por el contrario, sostiene que éste obedece a una lógica deductiva, que el conocimiento científico se basa en teorías y afirma que la labor de los hombres y mujeres de ciencias es formular proposiciones y sistemas de proposiciones, los cuales han de ser contrastados rigurosamente. Para Popper, toda proposición o sistema de proposiciones es científico en virtud de que puede ser falsado mediante lo que denominó experimentos cruciales.

Otra versión desde la lógica deductiva es la que propone Kuhn (1972), quien rechaza la idea de que los hombres y mujeres de ciencias formulan teorías para falsarlas después. Desde un estudio de la historia del conocimiento científico, propone las categorías de paradigma, paradigmas en competencia, crisis paradigmática, comunidad y revolución científica para explicar el desarrollo histórico de ese conocimiento. Un paradigma se convierte en una especie de modelo cuando la comunidad de especialistas acepta una teoría para la formula- ción y solución de problemas durante cierto tiempo, lo que denomina ciencia normal.

Lakatos (1983) critica las propuestas de Popper y de Kuhn, y propone que el conocimiento científico se desarrolla a partir de programas de investigación. Lakatos sustenta que todo programa de investigación está constituido por un núcleo firme, el cual está organizado por tres o cuatro leyes, o proposiciones centrales, y por un cinturón protector conformado por hipótesis auxiliares, que se constituirán en el modelo para diseñar contrastaciones en la búsqueda de apoyo empírico para el programa. El cinturón protector recibe las contrastaciones de las hipótesis auxiliares. Si estas son positivas, se dice que el programa científico de investigación se hace progresivo; si son negativas, el programa se hace regresivo.

De acuerdo con lo anterior, se examinó si en los respectivos documentos predomina una concepción de ciencia como conjunto de observaciones ateóricas que obedecen a la lógica acumulativa de descubrimientos, y que, por tanto, no se enclava en un trabajo sistemático de formación de comunidades de especialistas; hasta qué punto obedecen a una labor de individuos geniales; o, por el contrario, si se habla desde una versión deductivistaconstructivista. 


\section{Aspecto Epistemológico específico}

El estudio epistemológico específico introduce la necesidad de examinar de qué manera se abordan las teorías, los paradigmas, los programas de investigación científicos o los modelos científicos en la formación inicial de profesores de química. Examina también, desde la perspectiva de sus estructuras conceptuales y metodológicas, las limitaciones de los problemas que se formularon y resolvieron en su interior, de aquellos cuyas soluciones propuestas no resolvieron y que, por tanto, dan pie a la necesidad de formular otros, sustitutivos cuya aceptación por la respectiva comunidad de especialis- tas obedeció a la misma dinámica.

Esta especificidad epistemológica remite indispensablemente al recuento histórico de la didáctica empleada, cuya obligatoriedad de enseñanza y de aprendizaje no podía tener otros propósitos que los meramente formativos.

Lo anterior es de importancia para responder a los siguientes cuestionamientos: ¿qué versión de la química están aprendiendo los profesores en formación? ¿Qué representaciones construyen los futuros docentes de la enseñanza de la química?

\section{La Química como Ciencia}

Desde una versión epistemológica constructivista, la química como ciencia se ubica dentro de una lógica deductiva, ya que los hombres construyen representaciones de sí mismos, de la sociedad y de la naturaleza, elaborando significados, formas de significar y de actuar (Gallego Badillo y Pérez Miranda, 1997). Así los químicos han construido representaciones específicas de sus objetos de conocimiento, edificando estructuras conceptuales y metodológicas que conforman los modelos científicos con los que y a partir de los cuales trabajan.
Desde la propuesta de Lakatos, se considera que la química ha evolucionado a partir de programas de investigación científica, ya que las comunidades de los químicos han creado categorías como la existencia de objetos de conocimiento e investigación, de cuerpos de conocimiento y de experimentos propios de la química (Gallego Badillo, Pérez Miranda y Torres de Gallego, 1995). Se propone que el objeto de conocimiento en química son las interacciones de sustancia-sustancia y sustancia-energía. La sustancialidad es entendida como una construcción ma- 
temática, expresada en longitudes, energía de enlace, reactividad y geometría molecular. Los cuerpos de conocimiento de los programas de investigación científica de los químicos se sustentan desde las teorías de la molecularidad de la interacción sustancia-sustancia y sustancia-energía. Se entiende por molecularidad, una propuesta que explica y describe los ordenamientos, las transformaciones, las regulaciones y los controles de los enlaces entre átomos, vistos desde una teoría de enlace, válida para la comunidad científica en el momento.

Desde la propuesta de Lakatos, el núcleo firme del programa de investigación científica en química lo conforman la ley de la conservación de los pesos, la de las proporciones definidas y la de las proporciones múltiples. El cinturón protector lo conforman las hipótesis deducibles del núcleo firme, las que se someten a contrastación empírica, originando así los experimentos.
El experimento químico es el medio por el cual se corroboran positiva o negativamente las hipótesis que estructuran el cinturón protector. Cuando los resultados son negativos, el cinturón puede ser parcial o totalmente modificado con la firme intención de proteger el núcleo firme que sustenta el programa de investigación.

Planteado lo anterior, se propone en este trabajo que el estatuto de cientificidad de la química se le otorgue desde los presupuestos de programa de investigación científica, y se afirma que la química es una construcción de una comunidad preocupada por resolver los problemas que se presentan en esta ciencia. Desde esta propuesta se indagó si los programas de formación inicial de profesores de química, en especial los documentos que sustentan éstos, tomaban alguna posición para argumentar la química como ciencia.

\section{La Ciencia de enseñar Ciencias}

Una de las consecuencias de la "revolución epistemológica", ocurrida en el siglo XX, fue la preocupación de un grupo de investigadores por cómo se impartía la educación en ciencias (Adúriz-Bravo e Izquierdo, 2002). Por esta razón deciden ocuparse del problema de la enseñanza de dichas ciencias.

Los resultados de esas investigaciones llevaron a la necesidad de dar estatuto de cientificidad a la didáctica de la naturaleza. Una primera propuesta, basada en la epistemología evolucionista de Toulmin (1977), la concibió como una empresa racional (Aliberas, Gutiérrez, e Izquierdo, 1989). Después se avanzó hacia la postulación de que la didáctica es una disciplina científica, teóricamente fundamentada (Gil y Col, 1999). En la actualidad se afirma que los especialistas han delimitado ya los campos de conocimiento e investigación (Gil Pérez, Carrascosa Alís y Martínez Terrades, 1999). Hay razones para pensar que hoy se cuen- 
ta con una ciencia de enseñar ciencias (Izquierdo y Sanmartí, 2001).

En relación con los campos de conocimiento e investigación estos son, entre otros, las concepciones alternativas de los estudiantes (Furió, 1996; Pozo, 1996); la resolución de problemas de lápiz y papel (Gil et al., 1999); las prácticas de laboratorio (Gil y Valdés, 1995); el diseño curricular (Gil et al., 1991); las relaciones ciencia-tecnología-sociedad y el papel del medio (Gil et al., 1991), y la formación inicial de docentes en ciencias experimentales (Gallego Badillo y Pérez Miranda, 2002; Mellado 1999).

Otros problemas fundamentales de los cuales se ocupan los didactas de las ciencias son los relacionados con la enseñabilidad (Gallego Badillo y Pérez Miranda, 1999) y las relaciones entre enseñanzas y aprendizajes. En cuanto al primero, el grupo de investigación Representaciones y Conceptos Científicos (Grupo IREC) es del parecer que se resuelve desde lo que algunos especialistas en educación matemática denominan transposición didáctica (Chevallard, 1997; Kang and Killpatrick, 1992), y los ingleses, recontextualización didáctica. Se trata de un proceso que convierte la ciencia que practican las comunidades de especialistas en ciencia escolar o escolarizada.

No obstante lo anterior, el problema de la enseñabilidad podría resolverse de otra forma, por lo que podrían darse versiones distintas. En todo caso, la enseñabilidad no podría partir de los textos escolares, porque lo que ellos contienen es ya ciencia didactizada.
En cuanto a la enseñanza como problema, parecería que no es formulable dentro de la concepción de la didáctica lineal y algorítmica, ni mucho menos desde la idea de que ella es la parte metódica de la pedagogía. Para tal efecto es necesario ubicarse conceptualmente en la didáctica como una disciplina teóricamente fundamentada, en la que la enseñanza, particularmente las estrategias, son de carácter metodológico.

En este orden de ideas, la enseñanza es formulada a partir de consideraciones no lineales y complejas (Hayles, 1982). En consecuencia, las estrategias tienen como concepto central la interacción; de ahí que sea necesario convertir el aula en un colectivo en el que el docente interactúa con el saber objeto de enseñanza y con los estudiantes, a la vez que propicia las interacciones entre ellos y los contenidos curriculares o unidades temáticas objeto de trabajo en el aula.

En la enseñanza así formulada, esos contenidos son propuestos como campos de trabajo cognoscitivo de todos los integrantes del colectivo aula, y pueden hacerse objeto de estudio desde la perspectiva de las competencias cognoscitivas (Gallego Badillo, 1999): interpretar, argumentar y proponer, soportados sobre el leer, escribir y hablar desde el lenguaje conceptual metodológico de cada unidad temática (Pérez Miranda, Gallego Badillo y Torres de Gallego, 2002).

En cuanto a las relaciones entre enseñanzas y aprendizajes, la formulación de éstas como problema exige 
la conceptualización de aquello que se quiere decir con aprendizaje. En efecto, dentro de la didáctica de las ciencias experimentales, éste ha sido pluralizado al criticar el aprendizaje memorístico y al pie de la letra (Ausubel, Novak y Haneisan, 1983). Se tiene, entonces, el significativo (Ausubel, Novak y Honeisan, 1983); el aprendizaje como cambio conceptual (Posner, Strike, Hewson and Gertzog, 1982); el del cambio conceptual y metodológico (Carrascosa y Gil, 1985); el aprendizaje como cambio conceptual, metodológico y actitudinal (Gené, 1991); y el del cambio conceptual, metodológico, actitudinal y axiológico (Gallego Badillo y Pérez Miranda, 1994), del cual se ha dicho que introduce la complejidad (Furió, 1996).
Hecha la anterior sustentación, el grupo IREC es del parecer que la didáctica de las ciencias es hoy la ciencia de enseñar ciencias, por lo que se considera que ésta no es la parte instrumental de la pedagogía, dado que en el campo internacional se encuentran investigaciones que abordan problemáticas de interés de la didáctica de las ciencias. Se debe recordar que en varios países de América y de Europa existen revistas especializadas en esta ciencia. Las comunidades dedicadas a esta disciplina celebran congresos, seminarios, etc., que han ido consolidando la didáctica como la ciencia de enseñar ciencias.

\section{Aspecto Pedagógico}

Como se sabe, la palabra pedagogía es introducida por primera vez, 1536, por Juan Calvino (1509-1564) en su obra Instituciones de la religión cristiana, en la que liga lo pedagógico a la educación y, especialmente, a la de los infantes.

En 1806 F. J. Herbert publica su obra Pedagogía general en la que, de conformidad con las críticas que se han hecho al respecto (Aguilla, 1968), además de fundar la cientificidad de la pedagogía en la filosofía y la psicología de su época, se centró en la enseñanza.

Admitido que los pedagogos en general se ocupan de estudiar la educación como un fenómeno cultural, social, económico y político, en este trabajo se parte de que los pedagogos de tales ciencias se ocupan de la educación, de lo educativo y de la educabilidad de las ciencias. 


\section{Acerca de lo Curricular}

Stenhouse (1991) considera que el currículo es el medio por el cual el profesor es invitado a probar ideas y alternativas en su ejercicio profesional; también es una vía para que adquiera conocimientos y aprenda sobre la naturaleza del mismo. El modelo curricular que propone este autor está ligado a un proceso de investigación y desarrollo del docente, buscando la reflexión en el ejercicio de su acción educativa, en que las ideas pedagógicas y didácticas han de probarse en la praxis con el fin de transformar las concepciones de los profesores.

Lundgren (1992) define el currículo como la solución necesaria del problema de la representación y el problema de la representación como el objeto del discurso pedagógico y didáctico. El problema de la repre- sentación se presenta cuando los procesos de producción se separan de la reproducción. Esta separación se presenta cuando estos dos procesos obedecen a un sistema lineal y continuo.

Otra propuesta de currículo invoca la versión de Nelson Ernesto y López Jiménez (1996), que proponen un currículo con un enfoque investigativo cualitativo y etnográfico y rechazan el currículo centrado en planes de estudio (asignaturas). Para estos autores el desarrollo del currículo está ligado a la evolución históricosocial de la humanidad y, paralelo a éste, el proceso educativo.

Las referencias anteriores sobre currículo sirvieron de base para analizar cuáles son las concepciones que al respecto dominan en los programas o proyectos curriculares de formación de profesores en química.

\section{Metodología}

De conformidad con la intencionalidad precisada y desde la revisión bibliográfica exigida, de acuerdo con el trabajo de grado aquí relacionado, se optó por una dirección metodológica de la cual se da cuenta a continuación.

Planteado el marco conceptual que orientó el presente trabajo, se procedió al análisis documental que sustenta la formación inicial de profesores de química tanto en la Universidad Pedagógica Nacional como en la Universidad Distrital Francisco
José de Caldas. Inicialmente se efectuó una lectura de los documentos, sin ningún propósito de indagar por las concepciones. Sólo se buscó información acerca de la propuesta de los dos programas de licenciatura en química. Una segunda fase fue la aplicación de las matrices a lo epistemológico, lo didáctico y lo pedagógico. Estas matrices (ver anexo) se denominaron de presencias y ausencias, y tienen su sustento conceptual y metodológico en el proyecto Los programas de formación inicial de licenciatura 
en ciencias experimentales: los acreditados por el CNA (Gallego Badillo, y Pérez Miranda, 2002).

Estas matrices están conformadas por afirmaciones que se desprenden del marco conceptual. Con una segunda lectura se procedió a buscar la presencia o ausencia de las mismas, es decir, si las afirmaciones se encontraban o no en cada uno de los documentos. En caso positivo, se concluye que hay una presencia; en el negativo, una ausencia. Las matrices, una para lo epistemológico, otra para lo didáctico y la tercera para lo pedagógico, estarán conformadas por una serie de afirmaciones que indicarán tendencias en los campos anotados; por tanto en la lectura de los documentos se buscarán tales afirmaciones.

\section{Resultados y ANÁlisis}

Planteado el marco conceptual y metodológico que orientó la investigación en referencia, se procedió a la aplicación de las matrices para la recolección y sistematización de la información en cuanto a las concepciones epistemológicas, pedagógicas y didácticas.

La recolección de la información se efectuó dentro de una metodología tipo análisis documental. Para la recolección y el análisis de la información requerida se emplearon las tres matrices diseñadas dentro de la línea de investigación (ver anexo) Formación inicial y continua de profesores de ciencias, del grupo IREC.

Las matrices se derivan de los fundamentos conceptuales de dicha línea y se hallan en sincronía metodológica con el marco de referencia. Como puede concluirse, estas matrices son específicas de tablas de presencias y de ausencias. Este nombre no hace alusión a la propuesta de Bacon (1979), ya que los fundamentos conceptuales aludidos se aproximan a las tendencias deductivistas-constructivistas.

Dado el carácter de las matrices y las bases de su diseño, la información con ellas recolectada se interpreta a la luz de lo establecido en el marco referencial en cuanto a lo epistemológico, didáctico y pedagógico.

Los proyectos en mención proponen que la ciencia no obedece a una linealidad de descubrimientos, sino a una construcción en comunidad que busca proponer explicaciones del mundo.

Cada proyecto estudiado formula su propia versión acerca de la didáctica. Un proyecto manifiesta que ésta se ocupa de los problemas relacionados con el enseñar y aprender a leer y a escribir; el otro propone que la didáctica se concibe como el resultado de un conjunto de postulados y de entramados teóricos y metodológicos con identidad epistémica propia. 
La enseñabilidad, para uno de los proyectos, es entendida como una construcción colectiva, en razón de que cada saber es enseñado por una comunidad de profesores. Para el otro es el núcleo del saber pedagógico.

Revisando el desarrollo de la pedagogía como disciplina, coinciden los dos proyectos que la pedagogía dis- pone de una serie de métodos para analizar e interpretar su objeto de estudio, la educación.

En el siguiente cuadro se presenta un análisis comparativo en cuanto a los fundamentos epistemológicos, didácticos y pedagógicos que direccionan la formación inicial de profesores de química de los dos programas estudiados.

\section{Análisis Comparativo Epistemológico}

\begin{tabular}{|l|l|}
\hline \multicolumn{1}{|c|}{ Proyecto 1 } & \multicolumn{1}{c|}{ Proyecto 2 } \\
\hline $\begin{array}{l}\text { Propone que la química no obedece a una linealidad de descubri- } \\
\text { mientos, sino que se desarrolla con los presupuestos de una lógica } \\
\text { deductiva. }\end{array}$ & $\begin{array}{l}\text { Desde una perspectiva constructivista, se considera que la ciencia } \\
\text { no se produce sobre una base objetiva de observaciones que persi- } \\
\text { gan comprobar las teorías sobre el mundo. }\end{array}$ \\
$\begin{array}{l}\text { La historia crítico-epistemológica de la química ha de mostrar cómo } \\
\text { la estructura lógico-disciplinar admitida es un producto histórico dado } \\
\text { por la productividad de la teoría, del paradigma o del programa de } \\
\text { investigación para su apoyo social y financiero. }\end{array}$ & $\begin{array}{l}\text { La ciencia no se elabora acumulativamente sobre teorías predece- } \\
\text { soras; por el contrario, ésta se valida en la posibilidad de falsación } \\
\text { de sus hipótesis, la competencia de programas de investigación, el } \\
\text { sostenimiento de paradigmas, los criterios de racionalidad o como } \\
\text { producto de competencia y evolución conceptual. }\end{array}$ \\
\hline
\end{tabular}

\section{Análisis Comparativo Didáctico}

La didáctica se ocupa de los problemas relacionados con el enseñar y aprender a leer y a escribir.
La didáctica se concibe como el resultado de un conjunto de postulados y de entramados teóricos y metodológicos con identidad epistémica propia, que permite elaborar nociones de realidad para el ámbito de la enseñanza de saberes y de otras formas de expresión cultural.

\section{Análisis Comparativo Didáctico}

La enseñabilidad es una construcción colectiva, en razón de que cada saber es enseñado por una comunidad de profesores.
La enseñabilidad de la química es el núcleo del saber pedagógico.

\section{Análisis Comparativo Pedagógico}

Es posible elaborar una teoría pedagógica desde la cual se asignen funciones a la educación. La teoría así construida se convierte en un modelo pedagógico dentro del cual se podrán resolver las siguientes preguntas: ¿para qué?, ¿con qué? y ¿cuándo educar?, en contraposición con las de qué y cómo enseñar.
Revisando el desarrollo de la pedagogía como disciplina, ésta dispone de una serie de métodos para analizar e interpretar su objeto de estudio, la educación. 
¿Qué es formar a un profesional de la química que se ocupe de los problemas pedagógicos y didácticos de esta ciencia? Es perfilar a un ser químico en el interior de una comunidad de especialistas, que se ocupa de la producción y explotación conceptual y metodológica de teorías químicas.
El futuro maestro debe aproximarse al debate sobre problemas pedagógicos y a la manera en que estos problemas se han abordado desde diferentes posiciones epistemológicas.
En cuanto a currículo, el proyecto se basa en lo expuesto por Stenhouse, que plantea el currículo como proyecto de investigación, También se apoya en la propuesta de Cesar Coll $(1986,1987)$, el cual considera el currículo como el proyecto educativo que incluye los aspectos curriculares en sentido limitado (objetivo, contenidos) y los aspectos instruccionales (relativos a cómo enseñar).
Asume el currículo como un programa de actividades investigativas, el cual surge al abordar problemas desde los cuales se intentan reconsiderar las ideas previas de los estudiantes y, por tanto, aproximarse a un aprendizaje significativo de la química.

\section{Conclusiones}

Analizados los documentos en mención, los autores del presente artículo se permiten afirmar lo siguiente:

Con la lectura y posterior aplicación de las matrices, se llegó a la conclusión de que los proyectos curriculares sustentan los dos programas de formación inicial de profesores de química desde una versión epistemológica, aparentemente constructivista; esto es, no se encuentra una argumentación sólida que sustente la versión epistemológica que orienta los programas estudiados.

En cuanto a la química, los proyectos enuncian que esta es una ciencia, pero ninguno de los dos justifica si la química se construye a partir de teorías, paradigmas, programas de investigación científica o modelos científicos.

Uno de los proyectos no justifica si la didáctica es una ciencia, ya que sólo enuncia que ésta se encarga de resolver los problemas de enseñar a leer y a escribir en química; por tanto se piensa que el proyecto asume la didáctica como la parte instrumental de la pedagogía. Hoy en día se sabe que la didáctica es una disciplina que está emergiendo, la cual se encarga de resolver aquellos problemas que se presentan en la enseñanza y aprendizaje de las ciencias experimentales.

El otro proyecto propone que la didáctica es una ciencia que posee unos postulados teóricos y que parte de una visión constructivista, pero no explica cuál es su objeto de estudio; por consiguiente no presenta un sustento conceptual ni metodológico de la didáctica de las ciencias como disciplina emergente.

A partir de la lectura y, posteriormente de la aplicación de las matrices, ambos proyectos coinciden en que el objeto de estudio de la pedagogía es la educación, por cuanto esta se encarga de resolver aquellos problemas que se presentan en ella.

En lo que respecta a lo curricular, no se evidencia una correspondencia lógica entre los presupuestos teóricos 
enunciados y la estructuración misma del currículo como concreción de esos presupuestos. En el documento se observa que lo conceptual no corresponde a lo metodológico, es decir, no hay una concordancia en- tre lo que se plantea y lo que se pretende hacer en la ejecución de los dos proyectos curriculares para la formación inicial de profesores de química.

\section{BibliografíA}

Adúriz Bravo, A. e Izquierdo A., (2002). Acerca de la didáctica de las ciencias como disciplina autónoma. Revista Electrónica de Enseñanza de las ciencias, 1 (3). En www.saum.uvigo.es/rec

Agulla. (1968). Sociología de la educación. Buenos Aires: Paidós.

Aliberas, J. Gutiérrez, R. e Izquierdo, M. (1989). La didáctica de las ciencias. Enseñanza de las Ciencias, 7 (3), 277-284.

Amador-Rodríguez R. Y. (2003). Estudio de dos Programas de Formación inicial de Profesores de química. Trabajo de grado. Bogotá: Universidad Pedagógica Nacional.

Ausubel, D., Novak, J. D. y Honeisan, (1983). Psicología educativa, un punto de vista cognoscitivo. Segunda edición. México: Editorial Trillas.

Bacon, F. (1979). Novum organom. México: Porrúa.

Carrascosa Allis, J. y Gil, Pérez. (1985). La metodología de la superficialidad y el aprendizaje de la ciencia. Enseñanza de las Ciencias, 3 (2), 113-120.

Chevallard, Y. (1997). La transposición didáctica. Aique Grupo editor.

Comte, A. (1984). Cursos de filosofía positiva. Barcelona: Orbis.

Dumas-Carré, A. Fruió, M. y Garret, R. (1990). Formación inicial del profesorado de ciencias en Francia, Inglaterra y Gales y España. Análisis de la organización de los estudios y nuevas tendencias. Enseñanza de las Ciencias, 8 (3), 274-281.

Ernesto, N. y Jiménez, L. (1986). Retos para la construcción curricular. Mesa redonda, Magisterio.

Furió Mas, C. (1994). Tendencias actuales en la formación del profesorado de ciencias. Enseñanza de las Ciencias, 12 (2), 188-199.

Furió Mas, C. (1996). Las concepciones alternativas del alumnado. Dos décadas de investigación. Resultados y tendencias. Alambique. Didáctica de las Ciencias Experimentales, 7, $7-17$.

Furió Mas, C. y Gil Pérez, D. (1989). La didáctica de las ciencias en la formación inicial del profesorado: Una orientación y un programa teóricamente fundamentado. Enseñanza de las Ciencias, 7 (3), 257-265.

Gallego Badillo, R. y Pérez Miranda, R. (1997). La enseñanza de las ciencias experimentales. Bogotá, Cooperativa Editorial Magisterio.

Gallego Badillo, R. y Pérez Miranda. R. (2002). Proyecto: Los programas de formación inicial de licenciatura en ciencias experimentales: los acreditados por el C.N.A. V convocatoria proyectos de investigación en educación y pedagogía. Universidad Pedagógica Nacional.

Gallego Badillo, R. y Pérez Miranda. R. (1999). Aprendibilidad-Enseñabilidad y Educabilidad de las ciencias experimen- 
tales. Revista Educación y Pedagogía Universidad de Antioquia. No 25: 89-117.

Gallego Badillo, R. y Pérez Miranda. R. (1994). Representaciones y conceptos científicos: Un programa de investigación. Universidad Pedagógica Nacional, Departamento de Química.

Gallego Badillo, R., Pérez Miranda. R. y Torres de Gallego. (1995). La químca como ciencia: una perspectiva constructivista. Química actual y futuro. 5 (1) 55-63.

Gallego Badillo, R. (1999). Las competencias cognoscitivas. Un punto de vista epistemológico, didáctico y pedagógico. Cooperativa editorial magisterio.

Gené, A. (1991). Cambio conceptual y metodológico en la enseñanza y el aprendizaje evolución de los seres vivos. Un ejemplo concreto. Enseñanza de las Ciencias, 9 (1), 26-27.

Gil Pérez, D., Carrascosa Alís, J. y Martínez Terradez, F. (1999). El surgimiento de la didáctica de las ciencias como campo específico de conocimientos. Revista Educación y Pedagogía Universidad de Antioquia. No 25: 15-65.

Gil, D., Furió, C., Valdés, P., Salinas, J., Martínez-Torregrosa, Guisasola, J., González, E., Dumas-Carré, A., Goffard, M. y Pessoa. (1999). ¿Tiene sentido seguir distinguiendo entre aprendizaje de conceptos, resolución de problemas de lápiz y papel y realización de prácticas de laboratorio?. Enseñanza de las ciencias 1999 17(3), 311-320

Gil, D., y Valdés, P. (1995). Un ejemplo de práctica de laboratorio como actividad investigadora, Alambique, 6, 93-102.

Gil Pérez, D. (1991). ¿Qué hemos de saber y saber hacer los profesores de ciencias?. Enseñanza de las Ciencias, 9 (1), 69-77.

Gil, D.; Belendez A.; Martín A. y MartínezTorregrosa J. (1991). La formación del pro- fesorado universitario de materias científicas: contra algunas ideas y comportamientos «de sentido común», Revista Interuniversitaria de Formación del Profesorado, 12, 43-48

González , E.; Arena, L.; Budde, C. De Longhi, A. L.; Ferreira, A. y Re, M. (1996). Cinco ejes para la discusión sobre la formación inicial y la capacitación de los docentes de ciencias. Revista de Enseñanza de la Física, 9(2), 75-83.

Hodson, D. (1985). Phylosophy of Science, Science and Science Education. Studies in Science Education, 12 (1), 25 - 27.

Hayles, N. K. (1982). La evolución del caos. Barcelona: Gedisa.

Izquierdo, M. y Sanmartí, N. (2001). Hablar y escribir para enseñar ciencias. Enseñanza de las Ciencias. Número Extra, VI Congreso.

Kang, W., y Kilpatrick, J. (1992). For The Learning of Mathematics. 12 (1). Publishing Association, White Rock, British Columbia, Canada.

Kuhn, T. S. (1972). La estructura de las revoluciones científicas. México, Fondo de Cultura Económica.

Lakatos, I. (1983). La metodología de los programas de investigación científica. Madrid, Alianza.

Lundgren, U. P. (1992). Teoría del curriculum y escolarización. Madrid, Morata.

Mellado Jiménez, V. (1999). La investigación sobre la formación del profesorado de ciencias experimentales. La didáctica de las ciencias. Tendencias actuales. Universidad Coruña. 45-76.

Mellado Jiménez, V. y González, T. (2000). La formación inicial del profesorado de ciencias. Didáctica de las ciencias experimentales. Alcoy, España: Marfil. 
Popper, K. (1962). La lógica de la investigación científica. Madrid: Tecnos.

Posner, G. J., Strike, K. A., Hewson, P. W., and Gertzog, W. A. (1982). Acocommodation of a scientific conception: towar a theory of conceptual change. Science Education, 66 (2), 211-227

Pozo. (1996). Las ideas del alumno sobre ciencias. De dónde vienen, a dónde van y... mientras tanto, qué hacemos con ellas. Alambique. Didáctica de las Ciencias Experimentales, 7, 18-26.

Stenhouse, L. (1991). Investigación y desarrollo del currículo. Madrid: Morata.

Toulmin, S. (1972). La comprensión humana. Madrid: Alianza Editorial.

\section{Anexo}

\section{Matrizepistemológica}

\section{Generalidades}

\begin{tabular}{|c|c|c|}
\hline Afirmaciones & Sí & No \\
\hline \multicolumn{3}{|l|}{ 1. Precisa la tendencia epistemológica del programa. } \\
\hline \multicolumn{3}{|l|}{ 2. Caracteriza las aproximaciones empiropositivistas. } \\
\hline \multicolumn{3}{|l|}{ 3. Identifica las racionalistas. } \\
\hline \multicolumn{3}{|l|}{ 4. Discurre sobre las deductivistas. } \\
\hline \multicolumn{3}{|l|}{ 5. Delimita las constructivistas. } \\
\hline \multicolumn{3}{|l|}{ 6. Asume una posición ecléctica. } \\
\hline \multicolumn{3}{|l|}{ 7. Establece las diferencias entre las aproximaciones anotadas. } \\
\hline \multicolumn{3}{|l|}{ 8. Destaca el papel de cada una de esas aproximaciones en la didáctica de la química. } \\
\hline \multicolumn{3}{|l|}{ 9. Discrimina entre una química desarrollada por individuos y otra por colectivos disciplinares. } \\
\hline 10. Justifica los estudios epistemológicos en la formación inicial del profesor de química. & & \\
\hline
\end{tabular}

\section{Especificidades}

\begin{tabular}{|c|c|c|}
\hline Afirmaciones & Sí & No \\
\hline 1. Afirma que el conocimiento químice & & \\
\hline 2. Le da primacía al método científico. & & \\
\hline 3. Admite la pluralidad metodológica. & & \\
\hline
\end{tabular}


Tecné, Episteme y Didaxis No. 16, 2004 - páginas 64 - 83

Especificidades

\begin{tabular}{|c|c|c|}
\hline Afirmaciones & Sí & No \\
\hline 4. Puntualiza que la química está conformada por teorías, paradigmas, programas d & & \\
\hline \multicolumn{3}{|l|}{ 5. Explicita la aproximación epistemológica que orienta el programa. } \\
\hline \multicolumn{3}{|l|}{ 6. Predomina la de teoría. } \\
\hline \multicolumn{3}{|l|}{ 7. Acoge la de paradigma. } \\
\hline \multicolumn{3}{|l|}{ 8. Hay preferencia por la categoría de programa de investigación. } \\
\hline \multicolumn{3}{|l|}{ 9. Adopta la de modelo científico. } \\
\hline \multicolumn{3}{|l|}{ 10. Se refiere a ellas de forma indistinta. } \\
\hline \multicolumn{3}{|l|}{ 11. Discurre en torno al carácter hipotético-deductivo de esas categorías. } \\
\hline \multicolumn{3}{|l|}{ 12. Precisa al papel del experimento en química. } \\
\hline \multicolumn{3}{|c|}{$\begin{array}{l}\text { 13. Se ocupa de la lógica interna de las estructuras conceptuales y metodológicas de teorías, paradigmas, programas } \\
\text { o modelos químicos. }\end{array}$} \\
\hline \multicolumn{3}{|l|}{ 14. Estipula el papel de la historia interna de la química. } \\
\hline \multicolumn{3}{|c|}{$\begin{array}{l}\text { 15. Analiza las razones por las cuales a lo largo de la historia, unas teorías, paradigmas o modelos han sido sustituidos } \\
\text { por otras o por otros. }\end{array}$} \\
\hline \multicolumn{3}{|l|}{ 16. Formula el conocimiento químico como lineal y acumulativo. } \\
\hline \multicolumn{3}{|c|}{ 17. Plantea el problema de la obsolescencia de teorías, paradigmas, programas o modelos químicos. } \\
\hline \multicolumn{3}{|l|}{ 18. Para el programa, el conocimiento químico se encuentra en la naturaleza. } \\
\hline \multicolumn{3}{|l|}{ 19. Afirma que las leyes en química se descubren. } \\
\hline 20. Destaca la importancia de las revistas científicas, especializadas en química. & & \\
\hline
\end{tabular}

\section{MATRZZDIDÁCTICA}

Generalidades

\begin{tabular}{|c|c|c|}
\hline Afirmaciones & Sí & No \\
\hline 1. Sostiene que la didáctica es el componente metódico de la pedagogía. & & \\
\hline 2. Asume la didáctica como el arte de enseñar. & & \\
\hline 3. Acepta que para enseñar química basta con dominarla. & & \\
\hline 4. Predomina la concepción instrumental lineal o algorítmica de la didáctica. & & \\
\hline
\end{tabular}




\begin{tabular}{|c|c|c|}
\hline Afirmaciones & Sí & No \\
\hline 5. Acoge la formulación de que la didáctica de la química es una disciplina teóricamente funda & & \\
\hline $\begin{array}{l}\text { 6. Plantea que primero hay que conocer la ciencia por enseñar, para después ocuparse de lo } \\
\text { enseñanza. }\end{array}$ & & \\
\hline 7. Afirma que el método científico es el adecuado para enseñar química. & & \\
\hline 8. Estipula la aproximación epistemológica que hace de la didáctica de la química, una discipl & & \\
\hline 9. Precisa las razones del carácter técnico de esa didáctica. & & \\
\hline 10. Hace un análisis crítico entre una didáctica instrumental y una conceptual y metodológica. & & \\
\hline
\end{tabular}

\section{Especificidades}

\begin{tabular}{|c|c|c|}
\hline Afirmaciones & Sí & No \\
\hline 1. Asume la formación inicial de profesores de química como una línea de investigación. & & \\
\hline 2. Delimita los problemas didácticos que acarrea esa formación. & & \\
\hline 3. Adopta la idea de que se aprende a enseñar química por observación e imitación. & & \\
\hline 4. Aborda la identificación y caracterización de las ideas previas de los estudiantes sobre la enseñanza de la química. & & \\
\hline $\begin{array}{l}\text { 5. Puntualiza el programa en las concepciones epistemológicas y didácticas de los docentes de las asignaturas de futura } \\
\text { docencia. }\end{array}$ & & \\
\hline 6. Existe una posición crítica en relación con los textos de enseñanza de la química. & & \\
\hline 7. Precisa la formación en las teorías o modelos químicos desde análisis histórico-epistemológicos específicos. & & \\
\hline 8. Propone la revisión crítica de los originales de las teorías o modelos, como estrategia de formación. & & \\
\hline 9. Discurre en relación con la idea de que el conocimiento químico en el aula, ha de ser construido colectivamente. & & \\
\hline $\begin{array}{l}\text { 10. Hace hincapié en la incidencia de las concepciones epistemológicas y didácticas de los profesores de química en } \\
\text { su desempeño profesional. }\end{array}$ & & \\
\hline 11. Precisa una formación didáctica centrada en el estudio de las revistas especializadas, de esta disciplina científica. & & \\
\hline 12. Delimita conceptualmente y metodológicamente el problema de la enseñabilidad de las teorías o modelos químicos. & & \\
\hline 13. Confiere significado a la transposición o recontextualización didáctica de esas teorías o modelos. & & \\
\hline 14. Discute sobre las diferencias entre las química que practican los científicos y la química escolarizada. & & \\
\hline 15. Caracteriza didáctica y epistemológicamente las diversas clases de enseñanza. & & \\
\hline 16. Puntualiza en la enseñanza como un problema didáctico. & & \\
\hline
\end{tabular}




\begin{tabular}{|c|c|c|}
\hline Afirmaciones & Sí & No \\
\hline $\begin{array}{l}\text { 17. Conceptúa acerca de las relaciones entre esas diversas enseñanzas y los correspondientes aprendiz } \\
\text { esperados. }\end{array}$ & & \\
\hline 18. Discrimina las distintas formulaciones acerca del aprendizaje. & & \\
\hline 19. Procesa los marcos desde, los cuales se hacen emerger las diversas clases de enseñanza. & & \\
\hline 20. Fundamenta lo didáctico en referencias bibliográficas relativas a la didáctica como disciplina científica. & & \\
\hline
\end{tabular}

\section{Matriz peDAGógica}

\section{Generalidades}

\begin{tabular}{|c|c|c|}
\hline Afirmaciones & Sí & No \\
\hline \multicolumn{3}{|l|}{ 1. Caracteriza la pedagogía como una disciplina científica. } \\
\hline \multicolumn{3}{|l|}{ 2. Discurre en torno a las estructuras conceptuales y metodológicas de la pedagogía. } \\
\hline \multicolumn{3}{|l|}{ 3. Sostiene que el desempeño del pedagogo es un problema de aptitudes. } \\
\hline \multicolumn{3}{|l|}{ 4. Precisa los campos de investigación y conocimiento de los pedagogos. } \\
\hline \multicolumn{3}{|l|}{ 5. Adopta la mirada instrumental, técnica o algoritmica de la pedagogía. } \\
\hline \multicolumn{3}{|l|}{ 6. Discurre y caracteriza lo pedagógico de la química. } \\
\hline \multicolumn{3}{|l|}{ 7. Distingue entre saber pedagógico y saber didáctico. } \\
\hline \multicolumn{3}{|l|}{ 8. Establece diferencias entre pedagogía y enseñanza. } \\
\hline \multicolumn{3}{|l|}{$\begin{array}{l}\text { 9. Delimita conceptual y metodológicamente el problema de la formación inicial de un pedagogo de la } \\
\text { química. }\end{array}$} \\
\hline 10. Diferencia una pedagogía instrumental y una pedagogía científica. & & \\
\hline
\end{tabular}

\section{Especificidades}

\begin{tabular}{|c|c|c|}
\hline Afirmaciones & Sí & No \\
\hline $\begin{array}{l}\text { 1. Asume la formación inicial de un pedagogo de la química como cambio en sus concepciones alternativas } \\
\text { sobre la pedagogía. }\end{array}$ & & \\
\hline 2. Adopta una concepción sobre currículo. & & \\
\hline $\begin{array}{l}\text { 3. El currículo del programa es un proyecto de investigación en la línea de la formación inicial de profesores } \\
\text { de química. }\end{array}$ & & \\
\hline $\begin{array}{l}\text { 4. Posee referencias bibliográficas tomadas de revistas especializadas e indexadas en el campo de lo } \\
\text { pedagógico. }\end{array}$ & & \\
\hline
\end{tabular}


Estudio inicial de dos programas para la formación de licenciados en Química

\begin{tabular}{|c|c|c|}
\hline Afirmaciones & Sí & No \\
\hline 5. Está conceptual y metodológicamente fundamentado. & & \\
\hline 6. El plan de estudio se deriva de las hipótesis del proyecto curricular. & & \\
\hline 7. Precisa las correlaciones entre las asignaturas de futura docencia y las pedagógicas y didácticas. & & \\
\hline 8. Presenta la evaluación como un campo de conocimiento e investigación. & & \\
\hline 9. Distingue entre una evaluación pedagógica y didáctica y una meramente instrumental. & & \\
\hline 10. Analiza críticamente la evaluación habitual, practicada en las instituciones escolares. & & \\
\hline 11. En el proyecto curricular tanto las enseñanzas como los aprendizajes son objeto de evaluación. & & \\
\hline 12. Estipula los resultados que se constituirían en apoyo empírico del proyecto. & & \\
\hline 13. Fundamenta teóricamente la evaluación por grupos, como la individual. & & \\
\hline 14. Formula conceptual y metodológicamente el problema de la educación en química. & & \\
\hline 15. Diferencia esa educación de conformidad con los niveles y grados del sistema educativo. & & \\
\hline $\begin{array}{l}\text { 16. Establece relaciones entre las intencionalidades curriculares del sistema educativo y el proyecto, cultural, social, } \\
\text { político y económica. }\end{array}$ & & \\
\hline $\begin{array}{l}\text { 17. Relaciona la educación dentro del saber común y cotidiano y la educación en química que se lleva a cabo en las } \\
\text { instituciones escolares. }\end{array}$ & & \\
\hline 18. Estipula conceptual y metodológicamente lo educativo de la química. & & \\
\hline 19. Plantea rigurosamente el problema de la educabilidad en química. & & \\
\hline $\begin{array}{l}\text { 20. Discurre sobre la educación en, lo educativo de y la educabilidad que la química propician en términos de perte- } \\
\text { nencia social. }\end{array}$ & & \\
\hline
\end{tabular}

